\title{
Attosecond imaging
}

Cite this: Phys. Chem. Chem. Phys., 2014, 16, 2775

Received 29th August 2013, Accepted 14th November 2013 DOI: 10.1039/c3cp53659a

www.rsc.org/pccp

\author{
Marc J. J. Vrakking
}

The natural timescale for electron dynamics reaches down to the attosecond domain. Following the discovery of attosecond laser pulses, about a decade ago, attosecond science has developed into a vibrant, new research field, where the motion of single or multiple electrons and, in molecules, the coupling of electronic and nuclear motion, can be investigated, on attosecond to few-femtosecond timescales. Attosecond experiments require suitable observables. This review describes how "attosecond imaging", basing itself on kinetic energy and angle-resolved detection of photoelectrons and fragment ions using a velocity map imaging (VMI) spectrometer, has been exploited in a number of pump-probe experiments. The use of a VMI spectrometer in attosecond experiments has allowed the characterization of attosecond pulse trains and isolated attosecond pulses, the elucidation of continuum electron dynamics and wave packet interferometry in atomic photoionization and the observation of electron localization in dissociative molecular photoionization.

\section{Introduction}

The development of attosecond laser pulses $\left(1\right.$ as $\left.=10^{-18} \mathrm{~s}\right)$ in the first decade of this century has redefined the scope of a significant fraction of the research efforts devoted to ultrafast laser physics and chemistry. Whereas up to a decade ago ultrafast experiments on molecules were primarily concerned with time-resolving atomic motion within molecules in the course of photo-induced processes, ${ }^{1}$ the emergence of attosecond laser

Max-Born-Institut, Max Born Straße 2A, D-12489 Berlin, Germany.

E-mail: marc.vrakking@mbi-berlin.de

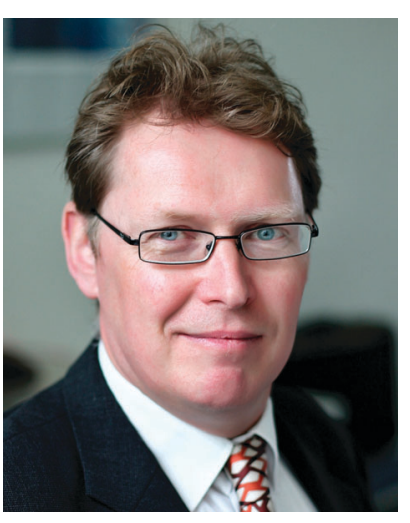

Marc J. J. Vrakking
Prof. Marc Vrakking obtained his PhD from the University of California at Berkeley in 1992. From 1997 to 2011 he led a group at FOM Institute AMOLF in Amsterdam, investigating the application of ultrashort (femtosecond and attosecond) extreme ultra-violet light pulses towards time-resolved studies of atomic and molecular dynamics. In March 2010 he became one of the directors of the Max Born Institute (MBI) in Berlin, in combination with a professorship at the FU Berlin. At the MBI he continues his research on novel ultrafast probes of electron dynamics and molecular rearrangement processes. pulses $^{2}$ has created unique possibilities for studying and controlling the electronic degrees of freedom as well as - in molecules the coupling of these degrees of freedom to the nuclear motion on attosecond to few-femtosecond timescales. Attosecond experiments offer novel opportunities both for the observation of ultrafast atomic and molecular dynamics and for its laser-driven control, exploiting both the short duration of currently available few-cycle and attosecond laser sources and the sensitivity of intense laser field driven processes to sub-cycle and hence attosecond-time scale variations of the laser electric field.

Besides the unprecedented time-resolution afforded by attosecond laser techniques, attosecond science offers unique possibilities for achieving spatial resolution. This is a natural consequence of the fact that attosecond pulses are tied to the use of short laser wavelengths in the extreme ultra-violet (XUV) and X-ray domain, since it is only here that the short optical periods needed for the synthesis of attosecond pulses are available. It follows that attosecond pulses exhibit photon energies that are substantially higher than atomic and molecular ionization potentials. This enables the ejection of energetic photoelectrons with, correspondingly, a short de Broglie wavelength $\left(\lambda_{\text {DeBroglie }}=\pi \sqrt{2 / E_{\text {kin }}}\right.$, so $\lambda_{\text {DeBroglie }}=1.0 \AA$ for a $150 \mathrm{eV}$ photoelectron). Consequently, the photoelectron angular distributions (PADs) accompanying the ionization of molecules by attosecond pulses contain structural information resulting from scattering of the ejected photoelectrons in the molecular frame, enriching the attosecond temporal resolution in the experiment with Angström spatial resolution. ${ }^{3}$

Around the world, intensive research efforts are ongoing, striving to use attosecond and, more generally, ultrashort XUV/X-ray techniques to achieve unprecedented temporal and 
spatial resolution. In this review, we will mostly focus on the temporal aspects, and will describe how multi-dimensional imaging techniques have allowed the rapid development of a range of successful attosecond pump-probe experiments that have started giving new insights into elementary electronic dynamics and the coupled motion of electrons and nuclei on attosecond to few-femtosecond timescales.

Attosecond pulses are inevitably formed in the process of high-harmonic generation (HHG). ${ }^{4}$ In this process, an intense femtosecond laser (typically a near-infrared (NIR) amplified Ti:Sapphire laser, with a central wavelength of about $800 \mathrm{~nm}$ ) is focused onto an atomic or molecular gas, at an intensity of $10^{14}-10^{15} \mathrm{~W} \mathrm{~cm}^{-2}$. As described by the three-step model proposed by Corkum ${ }^{5}$ and shown in Fig. 1, the high intensity leads to rapid ionization of the gas, followed by acceleration of the photoelectrons in the oscillatory laser field. During their oscillatory motion, a significant fraction of the electrons undergo re-collision with their parent ion, where recombination accompanied by the emission of coherent XUV/X-ray light can occur. Corkum's three-step-model successfully explained why HHG spectra extend to a high energy cut-off
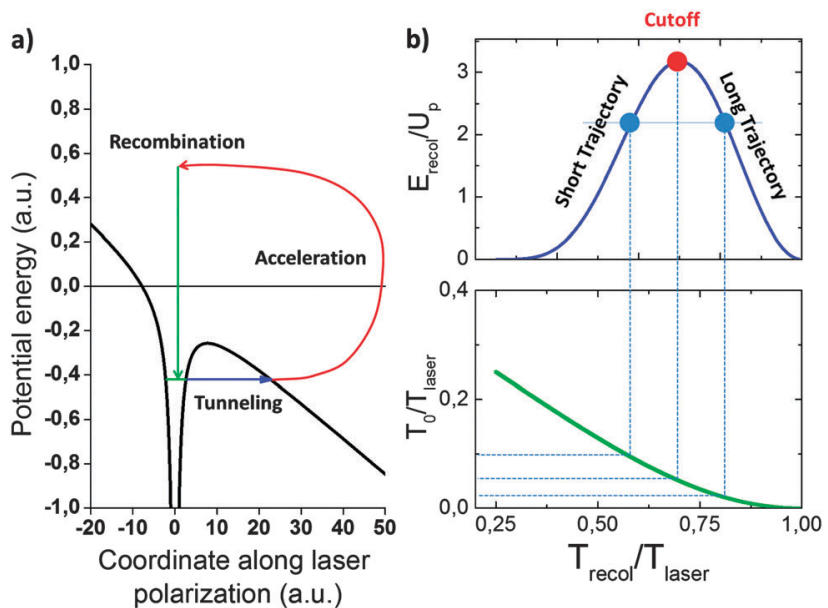

Fig. 1 (a) The three-step model of $\mathrm{HHG}^{5}$ Ionization (by tunneling through the Coulomb + laser field potential, blue arrow) launches an electron in the continuum, which is subsequently accelerated by the oscillatory NIR laser field. Along some of the trajectories (red arrow), the electron returns to the ionic core, and a recombination accompanied by the emission of coherent XUV/X-ray light occurs (green arrow). (b) The three-step model describes the trajectories of the electrons in the laser field, the relation between the time of ionization $T_{0}$ and the time of the electron-ion re-collision $T_{\text {recol, }}$ and the kinetic energy of the re-colliding electron that can be converted into photon energy. The maximum return energy of the electron is $3.17 U_{\mathrm{p}}$, defining the HHG cut-off (red dot). This energy is acquired by electrons that leave the atom around $T_{0} / T_{\text {laser }}=0.05$, i.e. shortly after a maximum in the NIR field. After acceleration in the laser field, these electrons return to the ion around $T_{\text {recol }} / T_{\text {laser }}=0.7$, i.e. after about $3 / 4$ of a laser cycle, near a zero-crossing of the laser field. Electron trajectories that are launched shortly after the cut-off trajectory return to the ion slightly earlier and are therefore called "short" trajectories, whereas ionization closer to $T_{0}=0$ leads to "long" trajectories. Contributions to the HHG from "long" and "short" trajectories can be separated on the basis of the XUV divergence and the phase-matching conditions, thereby allowing the clean production of attosecond pulses. given by $E_{\text {cut-off }}=\mathrm{IP}+3.17 U_{\mathrm{p}}$, where IP is the ionization potential of the atom/molecule used and $U_{\mathrm{p}}=\frac{I_{\text {laser }}}{4 \omega_{\text {laser }^{2}}{ }^{2}}$ is the ponderomotive energy, i.e. the average kinetic energy of a free electron in an oscillatory laser field with intensity $I_{\text {laser }}$ and laser frequency $\omega_{\text {laser. }}$. At an $800 \mathrm{~nm}$ laser wavelength and an intensity of $10^{14} \mathrm{~W} \mathrm{~cm}{ }^{-2}$, the ponderomotive energy is $6 \mathrm{eV}$, explaining why HHG produces XUV photons.

The three-step model can also be used to rationalize why HHG produces attosecond pulses. The first step in the threestep model, the ionization of the gas by multi-photon ionization, is a highly nonlinear process, which favors the maxima in the oscillatory electric field of the laser. This is all the more true when one considers that, under the conditions where most HHG experiments are performed, strong-field ionization is successfully described in terms of tunnel ionization, where the electric field of the laser distorts the Coulomb potential that binds the electron, and where the electron escapes this potential by tunneling through the barrier in the Coulomb + laser field potential (see Fig. 1). It follows that strong field ionization does not occur continuously during the entire optical period of the driving laser field, but only efficiently occurs during short intervals when the electric field is maximal. Given that the optical period of $800 \mathrm{~nm}$ light is only 2.7 femtoseconds long ( $1 \mathrm{fs}=10^{-15} \mathrm{~s}$ ), it follows that these intervals are very short, on the order of 100 as. During the acceleration in the laser field the ionized electron wave packet stays together, and consequently the recombination process - like the ionization - only occurs during a small fraction of the optical period, inevitably leading to attosecond pulses.

Still, to produce clean attosecond pulses several more steps are needed. First of all, the generated attosecond pulses co-propagate with the NIR laser that has generated them, meaning that a metal filter needs to be used that transmits the XUV/X-ray light, while blocking the NIR light. Secondly, every photon energy below $E_{\text {cut-off }}$ is produced twice per halfcycle of the driver laser, along what is commonly known as the "long" and "short" trajectories (see Fig. 1). Fortunately the divergence of the XUV light that is produced from both trajectories is rather different, meaning that in the far-field the attosecond pulses produced by the "short" trajectory can be selected by appropriate use of a spatial filter. Furthermore, HHG is a phase-matched process, which only reaches a measurable efficiency if the XUV emission from many emitters within an extended medium is in phase. HHG using the "long" and "short" trajectory is phase-matched under different conditions, providing another means of suppressing the unwanted attosecond pulses produced by the long trajectories. Finally, attosecond pulses are produced during every sufficiently intense half-cycle of the NIR laser, leading to the formation of attosecond pulse trains (APTs) rather than isolated attosecond pulses (IAPs). The formation of APTs is, at first sight, a problem for time-resolved pump-probe experiments, since it implies that the time where the attosecond pulse initiates or probes the ultrafast dynamics of interest is ill-defined. Still, in the years since their discovery, starting from experiments where attosecond 
phase-locking within an APT was demonstrated, ${ }^{6}$ scientists have learned how to perform meaningful attosecond experiments with APTs, and in this review we will encounter several examples. $^{7,8}$ At the same time, substantial efforts have been undertaken to produce IAPs using a range of methods, namely by working with very short NIR pulses and selecting the attosecond pulses from the cut-off region, ${ }^{9,10}$ by using an NIR pulse with a time-varying polarization and exploiting the fact that HHG requires the polarization to be linear, ${ }^{11}$ by using a two-color laser field, ${ }^{12}$ by working at high intensities where efficient phase-matching is only maintained during a small fraction of the NIR driving laser pulse, ${ }^{13}$ or using combinations of the above. Several examples of experiments utilizing IAPs will be discussed in this review. ${ }^{14,15}$

The experimental use of attosecond pulses still poses a number of severe challenges, explaining why almost two decades passed before the use of XUV radiation generated by HHG (discovered in the late $1980 \mathrm{~s}^{16,17}$ ) was mastered to a level that proliferation of the technique became possible. First of all, since XUV radiation is absorbed by all materials, experiments have to be entirely carried out in vacuum. Secondly, the development of efficient and high quality optics in the XUV range is very difficult. Thirdly, attosecond experiments pose extreme requirements on the stability of optical setups. Considering that a delay of 100 as corresponds to a distance of $30 \mathrm{~nm}$, and that in many attosecond setups the "pump" and "probe" lasers travel along separate paths over a distance of several meters, active stabilization of optical beam paths is often necessary in order to achieve the required time-resolution. Finally, the attosecond pulses obtained by HHG are so far very weak. A typical conversion efficiency in $\mathrm{HHG}$ is $10^{-6}$, meaning that mJ-level NIR driver pulses will be converted into nJ-level XUV/ $\mathrm{X}$-ray pulses. To make matters worse, the tailoring of the NIR pulse to enable the generation of IAPs induces further significant reductions in the efficiency, meaning that many of the first attosecond experiments were performed with pulse energies of just a few tens of pJ. These low pulse energies have consequences for the experiments that can be carried out. With sub-nJ pulse energies it is impossible, for example, to construct experimental setups where the probability that the atom/ molecule under investigation absorbs a photon from both a pump attosecond pulse and a probe attosecond pulse is sufficiently high. Correspondingly, the majority of attosecond experiments that have been performed up to now have been two-color XUV-NIR experiments where the attosecond time resolution that is inherent in the pulse duration of the attosecond pulse (APT or IAP) is combined with the use of the optical cycle of the NIR laser (as stated, $2.7 \mathrm{fs}$ at $800 \mathrm{~nm}$ ) as a clock with attosecond time-resolution. A number of ways in which this can be done will be discussed in this review. In the last few years, significant efforts are being undertaken to increase the pulse energy of attosecond pulses, and - in particular - the pulse energy of IAPs, in order to make XUV IAP pump-XUV IAP probe experiments possible. On the one hand, these approaches involve the use of larger scale laser systems delivering NIR pulses with several hundreds of mJ pulse energy. ${ }^{18,19}$ On the other hand, they involve the use of novel laser architectures like optical parametric chirped pulse amplification (OPCPA) that deliver NIR pulses with characteristics (in particular pulse durations) that are more favorable for generating IAPs than traditional Ti:Sapphire lasers. ${ }^{20}$ IAPs with pulse energies in the $100 \mathrm{~nJ}$ range have recently been reported ${ }^{19}$ and the first pumpprobe experiments using predominantly IAPs to both pump and probe attosecond dynamics have recently been published as well. ${ }^{21}$

As stated before, attosecond pulses involve photon energies that can ionize all neutral targets. As a result, until the recent emergence of attosecond transient absorption spectroscopy as a very popular and widely used method, ${ }^{22,23}$ the use of attosecond pulses has been largely based on measurements of charged particles that are formed in experiments. The first two experiments characterizing APTs and IAPs were based on measurements of the photoelectrons produced in two-color XUV + NIR ionization. In the case of APTs the dependence of the intensity of two-color photoelectron sidebands on the XUV-NIR delay was used to characterize the relative phase of a comb of harmonics, ${ }^{6}$ while IAPs were characterized by using somewhat stronger NIR fields, and making use of the same ponderomotive acceleration ("streaking") that underlies the HHG process: by measuring shifts and broadening/narrowing of the photoelectron spectrum as a function of XUV-NIR delay, ${ }^{24,25}$ a complete reconstruction of the IAP temporal shape is possible. ${ }^{11,26}$ The first successful application of attosecond pulses in an XUV + NIR pump-probe experiment, measuring the rate of Auger decay in $\mathrm{Kr}$ atoms, was also performed using attosecond streaking spectroscopy. ${ }^{27}$

The detectors used in these early experiments were timeof-flight photoelectron spectrometers, operated either under field-free conditions or in combination with a magnetic bottle. ${ }^{28}$ The advantages of using multi-dimensional detectors, such as velocity map imaging (VMI) detectors, ${ }^{29}$ giving access to both the kinetic energy and angular distribution of the ejected photoelectrons/photoions, were soon realized, ${ }^{30}$ and these days a substantial fraction of the experiments in attosecond science are carried out in this way. As detailed in other articles in this special issue, in a velocity map imaging detector photoelectrons/ ions produced in a (laser) ionization experiment are projected onto a two-dimensional detector, by applying an electric extraction field. The transport from the interaction region to the detector takes a finite time. Consequently, on the detector the particles are displaced from the center by an amount that is proportional to their velocity in the plane of the detector, explaining the name of the velocity map imaging technique. Using appropriate retrieval algorithms, the 3D velocity distribution of the detected particles can be retrieved, provided the experiment contains an axis of cylindrical symmetry (e.g. the laser polarization axis) in the plane of the detector.

In this review an overview of representative examples of "attosecond imaging”' experiments will be presented. We start in Section 2 with a discussion of the characterization of APTs using photoelectron velocity map imaging, ${ }^{30}$ and show how extensions of these experiments can be used to obtain information on the 
orbital from which the attosecond pulse removes an electron. ${ }^{7,31}$ Furthermore, under suitably chosen experimental conditions, the scattering of a re-colliding electron with the ionic core can be visualized $^{32}$ and electronic wave packets consisting of a superposition of Rydberg states can be reconstructed. ${ }^{14}$ Whereas Section 2 is largely concerned with atomic systems and continuum electrons, we turn to molecular systems and bound electrons in Section 3. Given that ponderomotive streaking of photoelectrons easily masks the bound electron dynamics of interest, these experiments use detection of - in particular fragment ions formed by dissociative ionization. We discuss experiments on control of electron localization in dissociative ionization of $\mathrm{D}_{2}$ using a carrier envelope phase (CEP) stable fewcycle laser pulse, ${ }^{33}$ which first allowed to infer the importance of attosecond time-scale laser-driven electron dynamics in molecules and discuss the extension of these experiments to XUVNIR attosecond pump-probe experiments, where the ionization of $\mathrm{D}_{2}$ is initiated using an IAP. ${ }^{15}$ While both of these experiments largely rely on dynamics in the $\mathrm{D}_{2}{ }^{+}$molecular ion, we also briefly discuss recent efforts to probe attosecond timescale electron dynamics in neutral systems. ${ }^{34}$ In Section 4 we conclude with a discussion of several other, complementary "attosecond imaging" methods, and present a brief outlook for future research.

\section{Attosecond photoelectron velocity map imaging}

As described in the introduction, HHG allows the conversion of NIR femtosecond laser pulses to coherent XUV/X-ray light, according to a mechanism that is commonly described by the three-step model. ${ }^{5}$ In a photon picture, the atom/molecule absorbs $N$ photons and emits a single photon with a photon energy $E=N \omega$. In the dipole approximation, parity considerations dictate that $N$ must be odd. Alternatively, one can consider that the attosecond pulses are produced twice per optical cycle, i.e. at an opposite sign of the electric field, also dictating that the spectrum is built up from odd harmonics. Correspondingly the time-dependent XUV electric field is given by

$$
E_{\mathrm{XUV}}(t)=\sum_{N_{\min }}^{N_{\max }}\left|E_{N}(t)\right| \cos \left(N \omega_{\text {laser }} t+\varphi_{N}\right)
$$

where $E_{N}(t)$ is an envelope function that describes the turn-on and turn-off of the harmonic radiation on femtosecond timescales. The time-dependent variations in $E_{\mathrm{XuV}}(t)$ are much too fast (in the $\mathrm{PHz}$ regime) to be measurable electronically. Therefore the characterization of APTs is based on measuring all the terms on the right-hand side of eqn (1), i.e. measuring $\left|E_{N}(t)\right|$ and the relative variations of $\varphi_{N}$ with $N$. Since we are predominantly interested in the attosecond duration of the XUV bursts, the time dependence of $\left|E_{N}(t)\right|$ is often neglected, meaning that its measurement reduces to a straightforward measurement of the HHG spectrum using an XUV spectrometer. Access to $\varphi_{N}$ can be obtained by interferometry, namely by recording sidebands in two-color XUV + NIR photoelectron spectra. ${ }^{6}$ For this reason the method is known as RABBITT (reconstruction of attosecond harmonic beating by interference of two-photon transitions). ${ }^{35}$

Fig. 2(a) shows the experimental arrangement that was used for the RABBITT measurements reported in ref. 30 . The output of a $50 \mathrm{~Hz} \mathrm{Ti:Sapphire} \mathrm{laser} \mathrm{system} \mathrm{(delivering} 70 \mathrm{fs}, 10 \mathrm{~mJ}$ laser pulses at $800 \mathrm{~nm}$ ) was split into an annular outer beam (with a diameter up to $20 \mathrm{~mm}$ ) and an inner beam with a diameter of $3 \mathrm{~mm}$, which were delayed with respect to each other by passing both of them through small pieces of glass that were tilted to slightly different angles. After the HHG process, the outer beam was blocked and the co-propagating harmonics and inner NIR beam crossed a skimmed atomic beam in the active region of a VMI spectrometer. Photoelectrons resulting from single-color XUV ionization and from two-color XUV \pm NIR ionization were both detected as a function of the delay $\Delta t$ between the outer and inner NIR beams, i.e. as a function of the XUV-NIR delay in the VMI experiment. Since the total XUV yield is maximal when the inner and outer NIR beams are in phase in the HHG gas jet, a measurement of the total XUV yield as a function of delay allowed the determination of the absolute time zero.

The intensity of the two-color sidebands oscillates as a function of the two-color delay and contains an interference term (see Fig. 2(b))

$$
S_{N+1} \sim \cos \left(2 \omega_{\text {laser }} \Delta t+\varphi_{N}-\varphi_{N+2}+\Delta \varphi_{\text {atomic }}\right)
$$

where $\Delta \varphi_{\text {atomic }}$ is a small atomic phase associated with the delay between the atomic ionization by $\mathrm{H}_{N}$ and $\mathrm{H}_{N+2} \cdot{ }^{36}$ As one can recognize from eqn (2), the sideband intensity undergoes two oscillations per NIR period. Fig. 2(c) shows two VMI images recorded during a typical RABBITT scan, where the left image was recorded at a delay where the XUV + NIR and XUV-NIR pathways interfered destructively (so that the sidebands are hardly visible), whereas the right image was recorded at a delay where both pathways interfered constructively. By measuring the sideband yield as a function of $\Delta t, \varphi_{N}-\varphi_{N+2}$ can be extracted. Performing this procedure for a number of sidebands allows determination of the relative variations of $\varphi_{N}$ with $N$ for a range of harmonics, and hence the reconstruction of the temporal shape of the XUV pulse (see eqn (1)). This is shown in Fig. 2(d) for APTs that were generated using $\mathrm{Ar}, \mathrm{Kr}$ and $\mathrm{Xe}$ as generating gas. In all three cases an APT is clearly observed, and in the case of Ar the duration of the individual attosecond pulses reaches 250 as. A remarkable observation in Fig. 2 is the delay between the APTs formed in Ar and $\mathrm{Kr}$ and those formed in Xe. According to the three-step model, APTs are expected to be formed at very similar times within the optical cycle of the driving laser field (around $T_{\text {recol }} / T_{\text {laser }}=0.7$, see Fig. 1 ). The observed differences between $\mathrm{Ar}, \mathrm{Kr}$ and Xe may be due to the fact that in $\mathrm{Ar}$ and $\mathrm{Kr}$ the HHG takes place in the tunneling regime, whereas in $\mathrm{Xe}$ it takes place in the multi-photon ionization regime, where resonant effects during the ionization can strongly affect the subsequent re-collision times. ${ }^{37}$

In the RABBITT characterization of an APT discussed above, the intensity of the NIR laser needs to be in the perturbative regime (i.e. $\sim 10^{11} \mathrm{~W} \mathrm{~cm}^{-2}$ ), in order to prevent contamination of the measured XUV \pm NIR sidebands by processes with a 
a)

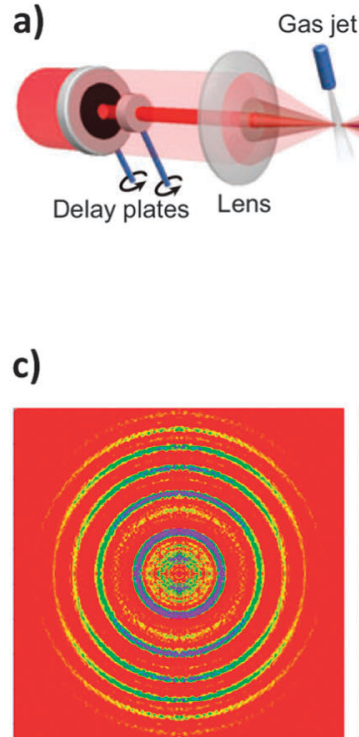

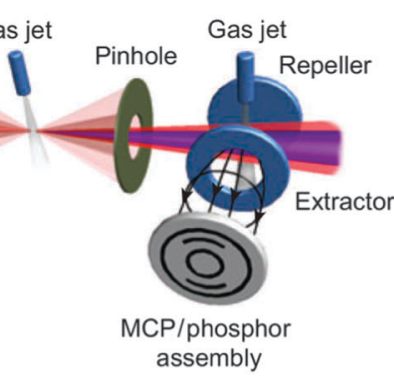

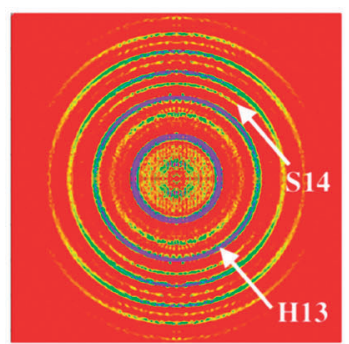

b)

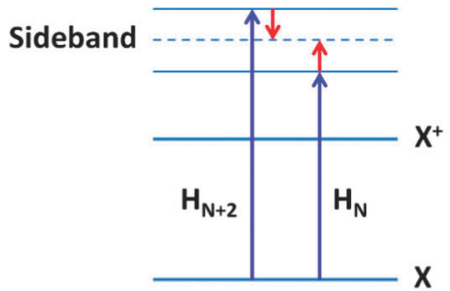

d)

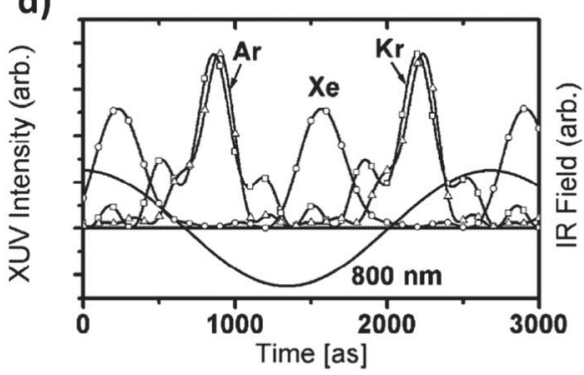

Fig. 2 (a) Experimental arrangement for the characterization of an attosecond pulse train (APT): attosecond pulses are generated in a gas jet, and propagate co-linearly with part of the NIR laser used to generate them towards a VMI detector, where photoelectrons from two-color XUV \pm NIR ionization are recorded. Prior to the HHG, the NIR beam is split into two parts, which can be delayed with respect to each other, namely an outer part that is predominantly responsible for the HHG and that is then blocked, and a central part that only weakly modifies the HHG process and that is used in the

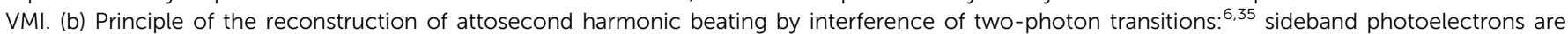
produced by two-color (XUV \pm NIR) ionization involving two adjacent harmonics $\mathrm{H}_{N}$ and $\mathrm{H}_{N+2}$; the two pathways interfere with each other and allow the extraction of the relative phase difference between the two harmonics. (c) Slice through the 3D velocity distribution measured for mixed-color (XUV \pm NIR) two-photon ionization of argon, where the XUV light was obtained by HHG in argon. The 3D velocity distribution contains sidebands due to twocolor (XUV $\pm N I R$ ) ionization involving adjacent harmonics. Arrows indicate photoelectron signals due to single-photon absorption of $\mathrm{H}_{13}$, resp. the sideband $\mathrm{S}_{14}$ involving interfering signals from $\mathrm{H}_{13}$ and $\mathrm{H}_{15}$. In the image on the left, the interference is destructive for most sidebands, whereas in the image on the right it is constructive. (d) Reconstructed APTs for HHG in Ar, Kr and Xe; in the case of Ar the full-width-at-half-maximum (FWHM) of the attosecond pulses is 250 as (adapted from ref. 30, copyright APS).

higher NIR non-linearity. When one wishes to characterize an IAP, where the XUV spectrum is continuous, the experimental requirements are very different. In this case sidebands cannot be observed, and the characterization is best performed in the "streaking" regime, where shifts and broadening/narrowing of the photoelectron spectrum are measured as a function of XUV-NIR delay. ${ }^{24,25}$ The streaking regime is conveniently described by considering that, following the creation of a photoelectron by an IAP at the time of ionization $t_{\text {ionization, }}$ the photoelectron acquires a momentum shift that is given by the time integral over the electric field of the NIR laser from $t_{\text {ionization }}$ to infinity:

$$
\Delta p=-\mathrm{e} \int_{t_{\text {ionization }}}^{\infty} E_{\text {laser }}\left(t^{\prime}\right) \mathrm{d} t^{\prime}=-\mathrm{e} A_{\text {laser }}\left(t_{\text {ionization }}\right)
$$

In other words, the photoelectron acquires a momentum shift that is proportional to the vector potential $A_{\text {laser }}\left(t_{\text {ionization }}\right)$ of the NIR laser at the time of ionization. Given the large bandwidth of an IAP, it is desirable that the associated energy shift is at least on the order of $5 \mathrm{eV}$. At a typical photoelectron kinetic energy of $54.4 \mathrm{eV}\left(E_{\mathrm{kin}}=2\right.$ a.u., i.e. $p=2$ a.u.) this requires $\Delta p \approx 0.09$ a.u., or, given that $E_{\text {laser }, 0}=\omega_{\text {laser }} A_{\text {laser }, 0}$, an NIR laser field with an intensity of appr. $10^{12} \mathrm{~W} \mathrm{~cm}^{-2}$.

Streaking measurements utilizing VMI were reported in ref. 38. In the experiment, Ne atoms were ionized by an IAP and subsequently ponderomotively streaked using an $800 \mathrm{~nm}$, NIR laser field whose peak intensity was estimated to be $10^{13} \mathrm{~W} \mathrm{~cm}^{-2}$. Fig. 3(a) shows the kinetic energy distributions of photoelectrons ejected upwards within an angle of $20^{\circ}$ from the common XUV/NIR polarization axis as a function of the XUV-NIR delay. Analysis of the experimental data using the FROG-CRAB algorithm ${ }^{26}$ led to the simulated streaking trace shown in Fig. 3(b) and the reconstructed IAP shown in Fig. 3(c), revealing the production of a 230 as pulse. The angular distributions that can be observed using VMI in the streaking regime have so far not extensively been used. However, they will become important in the next experiment to be discussed.

\section{Streaking of photoelectrons generated using APTs: attosecond wave packet interferometry}

While the characterization of APTs using RABBITT requires perturbative NIR intensities that are much lower than the intensities that are typically used in the streaking regime, very interesting experiments are also possible using APTs in this regime. The large ponderomotive momentum shifts induced by the NIR laser can create interference between photoelectrons that are ejected by different attosecond laser pulses, and this can provide information both on the NIR laser field and on the atom being ionized. A first example was reported in ref. 7 and is shown in Fig. 4. In these experiments an APT was generated by HHG in Ar, using a 35 fs Ti:Sapphire laser pulse. The APT was 

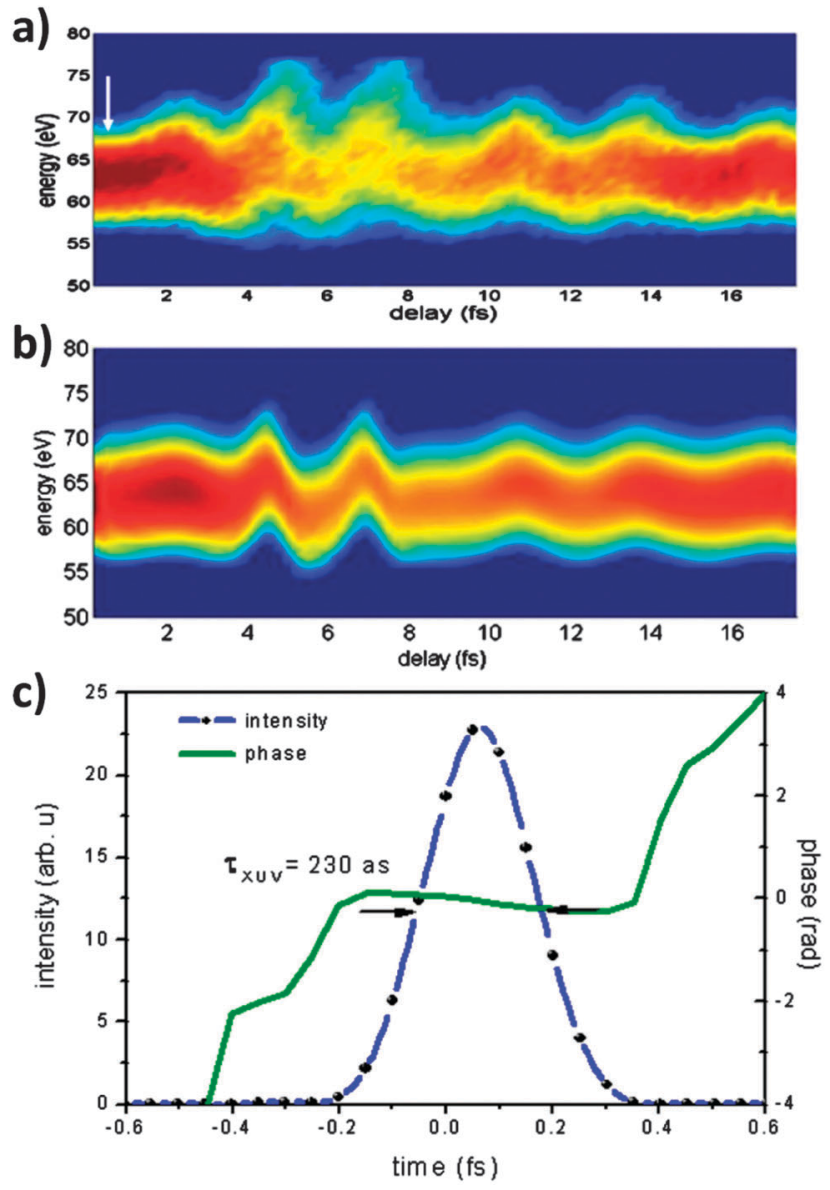

Fig. 3 (a) Streaking measurements, where an IAP was used to ionize $\mathrm{Ne}$ atoms in the presence of a NIR field with a peak intensity estimated at $10^{13} \mathrm{~W} \mathrm{~cm}^{-2}$. The measurement shows the kinetic energy distribution of photoelectrons emitted upwardly within $20^{\circ}$ of the XUV/NIR polarization axis, and recorded using a VMI spectrometer. (b) and (c) FROG-CRAB analysis ${ }^{26}$ of the measured streaking trace, showing the simulated streaking trace in (b) and the extracted XUV temporal intensity distribution (blue curve) and temporal phase (green curve) (courtesy of M. Kling, taken from ref. 38, copyright IOP).

co-linearly overlapped with an $\sim 2.5 \times 10^{13} \mathrm{~W} \mathrm{~cm}^{-2}$ NIR pulse, and VMI images were recorded as a function of the relative time delay between the APT and the NIR pulse. Fig. 4 shows two images that were obtained, corresponding to the case where the APT was synchronized to the zero-crossings of the NIR vector potential (left) and the case where the APT was synchronized to the maxima/minima of the NIR vector potential (right).

In the former case, when the APT is synchronized to zero crossings of the vector potential, there is no streaking, since the time integral of the laser electric field from the time of ionization is zero. Nevertheless, interesting interferences can be observed, due to differences in the phase accumulation of photoelectrons that are injected into the NIR laser field by different attosecond pulses within the APT. As explained in ref. 31, the interference pattern that is observed on the VMI detector can be understood by considering that (i) all observed photoelectrons result from the absorption of one harmonic photon and the absorption or stimulated emission of one or a)
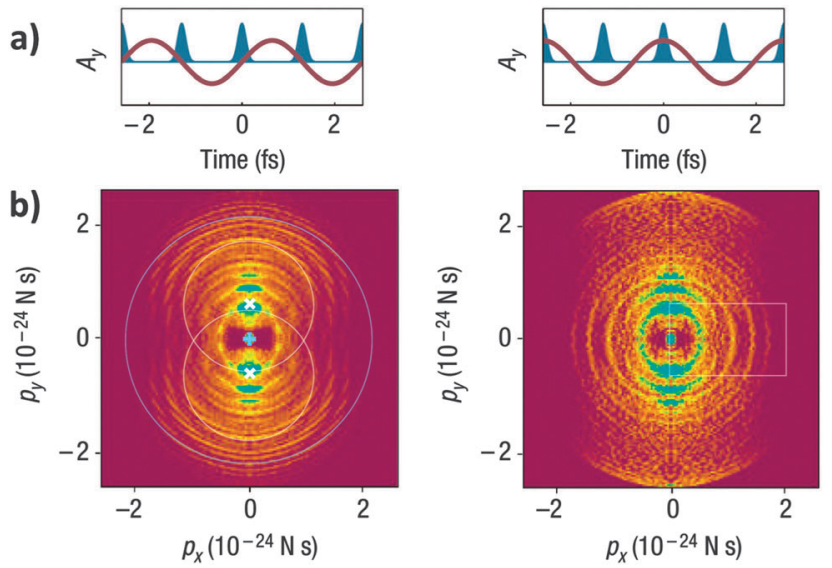

Fig. 4 (a) Two relative delays between the XUV APT and the vector potential $A_{\text {laser }}(t)$ of the co-propagating NIR field, where the attosecond pulses coincide with zero-crossings of $A_{\text {laser }}(t)$ (left), resp. with maxima of $A_{\text {laser }}(t)$ (right). (b) Reconstructed 3D velocity distributions resulting from a VMI measurement, for the two cases shown (taken from ref. 7, copyright Nature Publishing Group). In the case on the left, the measurement reveals a complicated interference pattern that allows one to determine the amplitude of the vector potential and hence the NIR laser intensity. In the case on the right ponderomotive streaking leads to interference (especially at right angles with respect to the laser polarization) between photoelectrons that are ejected in opposite directions along the laser polarization, therefore allowing determination of the symmetry of the continuum momentum wave function.

more NIR photons, and hence lie on concentric circles around the origin of the momentum map, and (ii) constructive interference between photoelectrons that are emitted by consecutive attosecond pulses requires that

$$
\begin{aligned}
& \frac{1}{2 m}\left[p_{x}^{2}+\left(p_{y}+(-1)^{k} \frac{2 \mathrm{e} A_{\text {laser }, 0}}{\pi}\right)^{2}\right] \\
& =(2 n+1) \frac{h}{2 \pi} \omega_{\text {laser }}-\mathrm{IP}+\left(\frac{8}{\pi^{2}}-1\right) U_{\mathrm{p}}
\end{aligned}
$$

where $p_{x}$ and $p_{y}$ are the photoelectron momentum perpendicular and parallel to the XUV/NIR laser polarization, $k$ and $n$ are integers, and IP is the ionization potential of the atom from which the photoelectron is removed. Eqn (4) describes concentric circles that are centered around two points $\left(p_{x}=0, p_{y}= \pm \frac{2 \mathrm{e} A_{\text {laser }, 0}}{\pi}\right)$ along the polarization axis. Hence, the observation of photoelectron wave packet interferences at this XUV-NIR delay provides a measurement of $A_{\text {laser, } 0}$, i.e. the vector potential (and hence the intensity) of the NIR field.

We may note that the condition where the attosecond pulses ionize atoms when the vector potential of the NIR field is zero is very similar to the condition that is encountered when a strong NIR field itself ionizes the atom by means of multi-photon or tunnel ionization. After all, as discussed in the introduction, such an ionization process favors the maxima of the laser electric field, i.e. the points where the vector potential is zero. Correspondingly, the interference patterns discussed above bear a resemblance to interference patterns 
observed in strong-field-induced above-threshold ionization (ATI), as confirmed by two recent publications. ${ }^{39,40}$

When the APT is synchronized to the maxima of the vector potential (right side of Fig. 4), normal streaking conditions apply, where the photoelectrons undergo momentum displacement given by the vector potential of the NIR at the time of their creation. Interestingly, this vector potential has the opposite sign for consecutive pulses in the APT. Consequently, if for ionization by one attosecond pulse $A\left(t_{\text {ionization }}\right)<0$ (accompanied by a momentum shift in the $+p_{y}$ direction), then for the next attosecond pulse $A\left(t_{\text {ionization }}\right)>0$ (accompanied by a momentum shift in the $-p_{y}$ direction). For a sufficiently high NIR laser intensity, the two opposing momentum shifts lead to interferences that are especially observable at right angles with respect to the laser polarization axis. In these interferences, symmetry properties of the photoelectron momentum wave functions manifest themselves. For example, as discussed in ref. 7 , for a momentum shift $\Delta p_{y}= \pm p_{\mathrm{NIR}}$, observable interferences at $p_{y}=0$ will measure the possible phase difference in the continuum wave function of the ejected photoelectron for momenta $\left(p_{x}, p_{y}=p_{\mathrm{NIR}}\right)$ and $\left(p_{x},-p_{\mathrm{NIR}}\right)$. If there is a sign change in the wave function between these momenta (as is the case for a p-orbital aligned along the $y$-axis), then this will be readily observable in the image. In ref. 7, XUV + NIR ionization of Ar atoms was studied, where both the $m=0$ and the $m= \pm 1$ components of the $l=1$ atomic ground state contributed. Comparison between experiment and theory illustrated that the latter components dominated.

\section{Observing re-collision using the attosecond quantum stroboscope}

In our description of RABBITT and attosecond streaking we have thus far only considered the interaction of the photoelectron with the NIR laser field. This is often a good approximation. However, this approximation breaks down if the photoelectron is formed with a small kinetic energy, or if the NIR field is very strong. Under these conditions, like in the HHG process, an NIR-driven re-collision of the electron with its parent ion can occur. This dynamics was investigated by Mauritsson et al. in ref. 32 and is shown in Fig. 5 . In the experiment, 300 as XUV pulses with a central energy of $24 \mathrm{eV}$ were used to ionize He atoms (IP = $24.6 \mathrm{eV}$ ), implying that a large fraction of the photoelectrons were formed with a small kinetic energy. A co-propagating NIR field with a peak intensity of $1.2 \times 10^{13} \mathrm{~W} \mathrm{~cm}^{-2}$ was used, and drove re-collision processes, which could clearly be observed in the VMI measurements shown in Fig. 5. We note that in order to maximize the utility of the experiment, special APTs were used, exhibiting only a single attosecond pulse per NIR cycle. The HHG process producing the attosecond pulses was carried out using the combination of an $800 \mathrm{~nm}$ NIR Ti:Sapphire laser and its $400 \mathrm{~nm}$ second harmonic. The presence of this $400 \mathrm{~nm}$ laser broke the symmetry in the HHG process, and led to a situation where there was only a single electric field maximum per optical cycle that led to the production of an attosecond pulse. Hence, contrary to the previously discussed wave packet interferometry results (Fig. 4 and ref. 7) there was only one attosecond pulse per NIR optical cycle and every attosecond pulse therefore encountered the same magnitude and polarity of the NIR laser field, prompting the authors to coin the term "attosecond quantum stroboscope" for this measurement technique. As a result of the use of an APT with just a single pulse per NIR optical cycle, the images shown in Fig. 5 reveal an up-down asymmetry, thereby providing a very transparent observation of the electron-ion re-collision process.

\section{Reconstructing bound state wave packets by quantum state holography}

Recently, a similar experiment was again carried out in $\mathrm{He},{ }^{14}$ but now with an IAP rather than an APT, and replacing the many-cycle NIR laser pulse used in ref. 32 by a few-cycle pulse. The important advantage is that - therefore - the time delay between the IAP exciting or ionizing the He atoms is not only defined on sub-NIR-cycle timescales, but can also be exploited over longer timescales. As we will discuss below, this allowed the reconstruction of a bound electron wave packet created by the part of the IAP spectrum below the He IP. The experiment can be viewed as the recording of a hologram, involving the
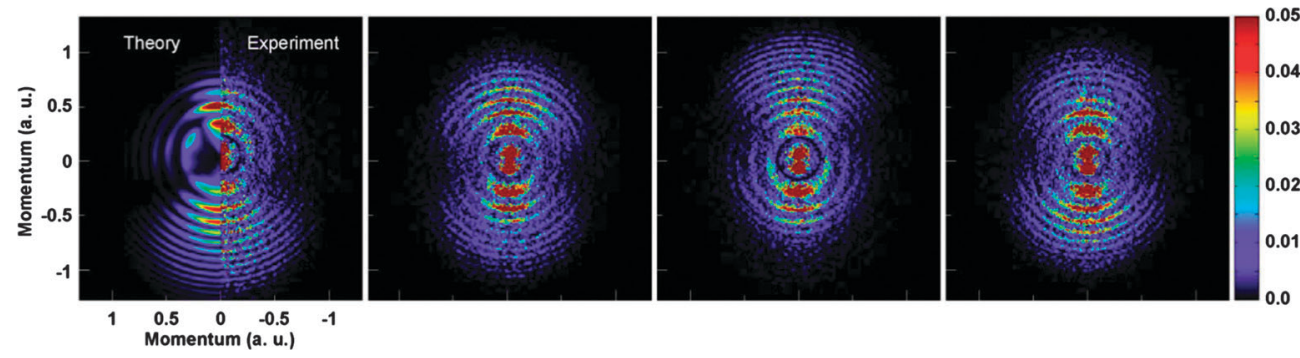

Fig. 5 Observations of electron-ion re-collision in two-color XUV + NIR ionization of He using an APT with a central energy (24 eV) close to the He ionization potential $(24.6 \mathrm{eV})$. Relatively slow photoelectrons resulting from ionization by an attosecond pulse re-collide with their parent ion under the influence of a moderately strong $\left(1.2 \times 10^{13} \mathrm{~W} \mathrm{~cm}^{-2}\right)$ NIR laser field. The four images were obtained for different relative delays between the XUV APT and the NIR pulse and, in each case, show a comparison between experimental results (right) and theoretical results obtained by numerically solving the timedependent Schrödinger equation (TDSE). As a result of the use of a two-color field in the HHG, APTs with just a single attosecond pulse per NIR optical cycle were produced, thereby enabling the observation of up/down asymmetries in the VMI images (courtesy of Dr Johan Mauritsson, taken from ref. 32 , copyright APS). 
a)

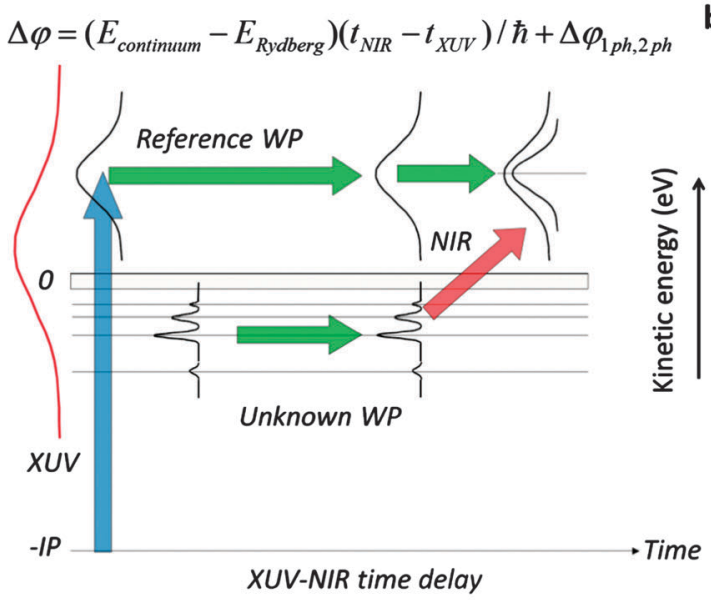

b)

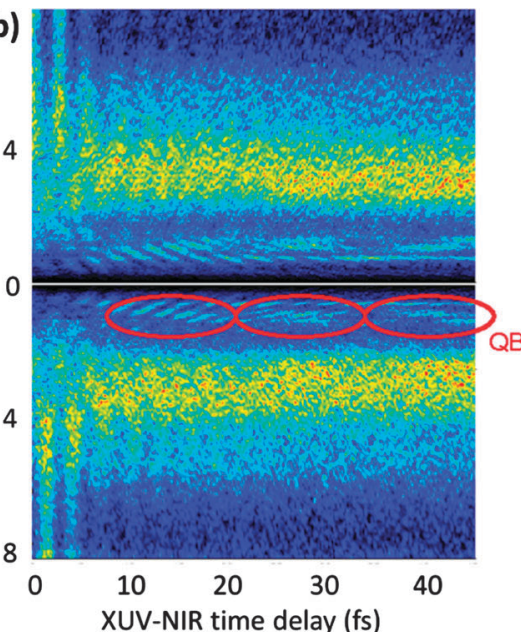

Fig. 6 (a) Holographic method for the complete characterization of bound electron wave packets. An IAP is used to ionize He, and creates a "reference" continuum electron wave packet. At a variable XUV-NIR time delay, a few-cycle NIR pulse is used to ionize an unknown Rydberg wave packet in He, thereby producing a "signal" continuum electron wave packet that interferes with the former reference wave packet on the VMI detector. (b) Kinetic energy distribution upwards and downwards along the XUV/NIR polarization axis as a function of the XUV-NIR time delay. By observing the holographic interference as a function of the XUV-NIR time delay, the energies, amplitudes and phases of the bound states contributing to the unknown Rydberg wave packet can be fully characterized. The method was first demonstrated in an experiment where the "unknown" Rydberg wave packet was produced by photo-excitation using the part of the IAP spectrum below the He IP (adapted from ref. 14, copyright APS).

interference between a "reference" electron wave packet resulting from ionization by the XUV IAP and a "signal" electron wave packet that results from XUV + NIR ionization.

A schematic of the experiment is shown in Fig. 6(a). He atoms are excited by an IAP that has part of its spectrum above the He IP, producing a "reference" continuum electron wave packet, and part of it below, producing a bound electron wave packet, i.e. a coherent superposition state containing contributions from a number of p-orbital Rydberg states. After the XUV IAP has passed, the continuum electron wave packet rapidly moves away from the atom, while the bound Rydberg wave packet displays radial oscillations. When, at a variable time delay, the NIR pulse is sent in, it influences the phase of the continuum electron wave packet (in a manner that can easily be calculated), without however changing the photoelectron kinetic energy, since optical fields cannot accelerate/decelerate free electrons. The bound wave packet is ionized by the NIR laser, producing a second "signal" continuum electron wave packet that interferes with the earlier "reference" electron wave packet on the VMI detector (see Fig. 6(b)), exploiting the high momentum resolution $(\Delta p / p \approx 0.01)$ that can be achieved. Considering a contribution at final photoelectron energy $E_{\text {continuum }}$ from the ionization of a Rydberg state with binding energy $E_{\text {Rydberg, }}$, the accumulated phase difference between the "reference" and "signal" wave packets may be written as

$\Delta \varphi\left(E_{\text {continuum }}, t_{\mathrm{NIR}}-t_{\mathrm{XUV}}\right)=\left(E_{\text {continuum }}-E_{\mathrm{Rydberg}}\right)\left(t_{\mathrm{NIR}}-t_{\mathrm{XUV}}\right)$

$$
+\Delta \varphi_{1 p h / 2 p h}
$$

where the last term allows for possible intrinsic phase differences in the XUV-only one-photon pathway and the XUV + NIR two-photon pathway, as well as a possible initial phase of the Rydberg state ionized. From eqn (5) it follows that Fourier transformation (FT) of the time-dependent photoelectron kinetic energy spectrum reveals both the energies $\left(E_{\text {Rydberg }}\right)$ as well as the populations and relative phases of the Rydberg wave packet populated by the IAP, representing a full characterization of this wave packet. ${ }^{14,41}$ We note that the experiment exploits not only the momentum resolution of the VMI detector, but the angular resolution as well. Decomposition of the photoelectron spectra (and the FT spectra derived from them) in terms of contributions of $P_{0}, P_{1}$ and $P_{2}$ Legendre polynomials allows a separate observation of the total signal $\left(P_{0}\right)$, the interference between XUV-only one-photon and XUV + NIR two-photon contributions $\left(P_{1}\right)$, and the observation of interference between different Rydberg states contained in the original bound state wave packet excited by the IAP $\left(P_{0}\right.$ and $\left.P_{2}\right)$. We furthermore note that the experiments reported in ref. 14 and 41 and Fig. 6 are to be understood as proof-of-principle experiments, characterizing a bound state wave packet that is a priori well known. The strength of the technique naturally lies in its ability to characterize unknown wave packets, such as electron wave packets arising through Auger decay or shake-up. We may expect experiments in this direction in the near future.

\section{Attosecond fragment ion velocity map imaging}

When attosecond pump-probe experiments are carried out on molecular systems, the same continuum electron dynamics that was discussed in the previous section in the context of atomic experiments will again occur. That is, in a strong NIR laser field photoelectrons will be streaked and in APT experiments with weaker NIR laser fields sidebands will be visible, as a result of two-color XUV \pm NIR interferences involving 
adjacent harmonics. Molecular RABBITT experiments have been carried out by Haessler and co-workers for $\mathrm{N}_{2}$, revealing the role of multiple continua and auto-ionizing resonances. ${ }^{42}$

Still, the use of angle- and/or energy-resolved photoelectron spectroscopy in attosecond pump-probe experiments on molecules is very challenging, given the substantial complexity of the photoelectron spectra that are encountered. This complexity is a consequence of the large intrinsic bandwidth of the attosecond pulses, which leads to resonant excitation and ionization processes involving a large number of electronic states. Furthermore, a relatively high intensity of the NIR pulses is typically needed, which makes it hard to work under conditions where the interaction with the NIR laser is strictly linear. The interaction with the NIR laser may even involve ponderomotive streaking of the outgoing photoelectrons, as discussed above, ${ }^{7}$ or may lead to transient Stark shifts which cause shifts of the observable photoelectron peaks. Realistically, we may therefore expect that widespread exploitation of angle- and energyresolved photoelectron measurements will require the further development of XUV IAP pump-XUV IAP probe experiments, ${ }^{21}$ or, in the case of two-color XUV + NIR experiments, the successful implementation of experimental protocols combining coincident detection of both the ion(s) and the electron(s) produced in a single pumpprobe event, ${ }^{43-45}$ in order to obtain more highly differential data.

Attosecond experiments on electron dynamics in molecular systems have so far focused on the detection of fragment ions. The first pump-probe experiment using attosecond laser pulses was published in $2010,{ }^{15}$ and was preceded by earlier experiments illustrating the possibility of driving and observing attosecond time-scale electron dynamics in small molecules. ${ }^{33,46}$ Rather than attosecond pulses, these earlier experiments used carrier envelope phase (CEP)-stable few-cycle NIR laser pulses.

Quite generally, the electric field of a femtosecond laser pulse can be written as

$$
E(t)=\left|E_{0}(t)\right| \cos \left(\omega_{\text {laser }} t+\varphi_{\mathrm{CEP}}\right)
$$

where $E_{0}(t)$ describes the pulse envelope (and possible timedependent changes in the carrier frequency of the pulse, which is assumed to have a maximum at $t=0$ ) and $\varphi_{\mathrm{CEP}}$ is the carrier envelope offset phase, which describes the phase offset between the maxima and minima in the rapid oscillations of the laser electric field and the maximum of the pulse envelope. For $\varphi_{\mathrm{CEP}}=0$, the maximum of the pulse envelope and one of the maxima of the laser electric field coincide ("cosine pulse"), whereas for $\varphi_{\mathrm{CEP}}=\pi / 2$ the maximum of the pulse envelope coincides with a zero-crossing of the electric field ("sine pulse"). Within the last decade, femtosecond laser systems with a controlled and reproducible $\varphi_{\mathrm{CEP}}$ have become common, and have played a major role in the development of attosecond science, given the fact that the controlled generation of IAPs requires that $\varphi_{\text {CEP }}$ is stable. ${ }^{47}$

In atomic physics, it was shown even before the development of CEP-stable femtosecond amplifiers that $\varphi_{\text {CEP }}$ plays an important role, allowing one to control the direction of emission of photoelectrons in strong-field ionization. ${ }^{48} \mathrm{~A}$ similar directionality was at the basis of the first observation of attosecond time-scale electron dynamics in a molecule. In the experiment, $\mathrm{D}_{2}$ molecules were dissociatively ionized using a CEP-stable, 5 fs long, $10^{14} \mathrm{~W} \mathrm{~cm}^{-2}$, $800 \mathrm{~nm}$ laser pulse. ${ }^{33}$ The initial dynamics in the molecule bears a considerable similarity to our earlier description of the three-step model (see Fig. 7a), namely ionization followed by acceleration of the freed electron in the oscillatory laser field leading to an electron-ion re-collision. However, in this case the focus of the experiment was on another possible outcome of the re-collision event, namely excitation of the $\mathrm{D}_{2}{ }^{+}$molecular ion from the initially formed $1 \mathrm{~s} \sigma_{\mathrm{g}}$ ground state to the repulsive $2 \mathrm{p} \sigma_{\mathrm{u}}$ first excited state. The proof for this dynamics was the observation of $\mathrm{D}^{+}$ ions with a high kinetic energy (up to $12 \mathrm{eV}$ ) and the dependence of these signals on the polarization state of the NIR laser.

Once the re-collision excites the molecular ion to the $2 \mathrm{p} \sigma_{\mathrm{u}}$ state, the fate of the molecule is - inevitably - dissociation.
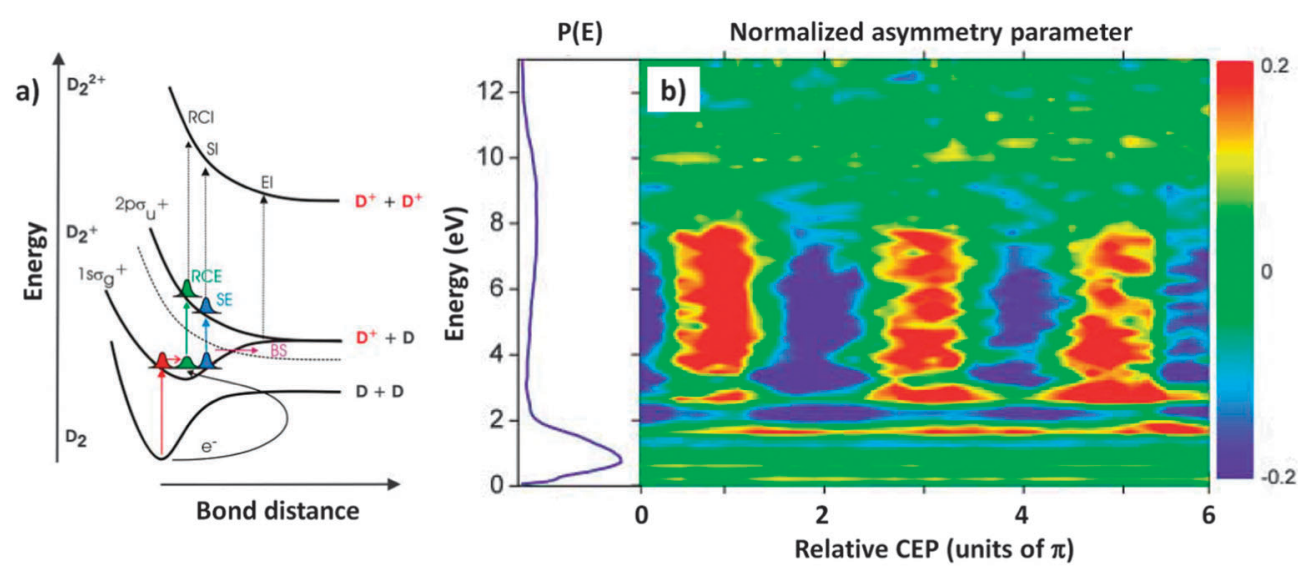

Fig. 7 (a) Potential energy curves of $D_{2}$ and $D_{2}{ }^{+}$, illustrating possible ionization mechanisms under the influence of a strong NIR field. Following ionization of the neutral molecule by multi-photon absorption (red arrow), populating the $1 \mathrm{~s} \sigma_{\mathrm{g}} \mathrm{D}_{2}{ }^{+}$ground state, further excitation to the $2 p \sigma_{\mathrm{u}}$ state is possible by means of a re-collision excitation (RCE, green arrow) or by means of a sequential excitation (SE, blue arrow) involving the absorption of one or more NIR photons; at higher IR intensities, Coulomb explosion is possible as well. (b) $\mathrm{D}^{+}$kinetic energy distribution $P(E)$ (left) and normalized asymmetry parameter $\left[P(E)_{\text {left }}-P(E)_{\text {right }}\right] /\left[P(E)_{\text {left }}+P(E)_{\text {right }}\right.$, as a function of the $\mathrm{D}^{+}$kinetic energy and the relative CEP of the few-cycle NIR pulse. The experiment reveals attosecond time-scale electron localization under the control of the CEP (adapted from ref. 33, copyright AAAS). 
However, given that the $2 \mathrm{p} \sigma_{\mathrm{u}}$ state has a well-defined ungerade parity, this dissociation will just as often lead to a $\mathrm{D}^{+}$ion that is flying right along the laser polarization axis as to a $\mathrm{D}^{+}$ion flying left along the laser polarization axis. In the experiment, the $\mathrm{D}^{+}$ ions were measured using a VMI detector. Remarkably, it was observed that the number of $\mathrm{D}^{+}$ions flying right and left could be different, and moreover, that $\varphi_{\mathrm{CEP}}$ controlled which direction was preferred. The main result is shown in Fig. 7b, which shows the normalized asymmetry defined as

$A\left(E_{\mathrm{kin}, \mathrm{D}^{+}}\right)=\left[P\left(E_{\mathrm{kin}, \mathrm{D}^{+}}\right)_{\text {left }}-P\left(E_{\mathrm{kin}, \mathrm{D}^{+}}\right)_{\mathrm{right}}\right] /\left[P\left(E_{\mathrm{kin}, \mathrm{D}^{+}}\right)_{\text {left }}+P\left(E_{\mathrm{kin}, \mathrm{D}^{+}}\right)_{\text {right }}\right]$

as a function of the kinetic energy of the $\mathrm{D}^{+}$fragments $E_{\mathrm{kin}, \mathrm{D}^{+}}$ and as a function of $\varphi_{\mathrm{CEP}}$. In order to determine the probabilities $P\left(E_{\mathrm{kin}, \mathrm{D}^{+}}\right)_{\text {left }}$ and $P\left(E_{\mathrm{kin}, \mathrm{D}^{+}}\right)_{\text {right }}$, the left and the right part of $\mathrm{D}^{+}$VMI images were integrated within $30^{\circ}$ of the polarization axis of the NIR laser. Fig. 7b shows clear oscillations of $A\left(E_{\mathrm{kin}, \mathrm{D}^{+}}\right)$as a function of $\varphi_{\mathrm{CEP}}$ for kinetic energies between 4 and $8 \mathrm{eV}$, where a shift of $\varphi_{\mathrm{CEP}}$ by $\pi$ leads to a reversal of the asymmetry.

The mechanism for this asymmetry is the laser-driven localization of the remaining electron in the dissociating $\mathrm{D}_{2}{ }^{+}$that relies on a dipole coupling between the $2 \mathrm{p} \sigma_{\mathrm{u}}$ and $1 \mathrm{~s} \sigma_{\mathrm{g}}$ states. As the molecule dissociates on the $2 \mathrm{p} \sigma_{\mathrm{u}}$ curve, it encounters a region where the de-excitation from the $2 \mathrm{p} \sigma_{\mathrm{u}}$ to the $1 \mathrm{~s} \sigma_{\mathrm{g}}$ state is a near-resonant process. Since dissociation of the $\mathrm{D}_{2}^{+}$molecular ion is so rapid, the molecule encounters this region within a few femtoseconds after the re-collision excitation to the $2 p \sigma_{\mathrm{u}}$ state. Consequently, the tail of the few-cycle NIR pulse is intense enough to de-excite a substantial fraction of the wave packet to the $1 \mathrm{~s} \sigma_{\mathrm{g}}$ state. The molecules that are eventually detected (via a measurement of the $\mathrm{D}^{+}$fragment on the VMI detector) are then in a coherent superposition of the $1 \mathrm{~s} \sigma_{\mathrm{g}}$ and $2 \mathrm{p} \sigma_{\mathrm{u}}$ states. This coherent superposition state does not have a well-defined parity, and hence the fraction of dissociating molecules where the $\mathrm{D}^{+}$ ion is detected on the right (in other words, where the remaining electron is localized on the left $\mathrm{D}^{+}$ion) can be different from the number of $\mathrm{D}^{+}$ions detected on the left (where the remaining electron is localized on the right). The importance of the attosecond time-scale laser-driven dynamics can be understood in the time-domain, by considering that the NIR laser drives the electron that remains in the dissociating $\mathrm{D}_{2}^{+}$ion back-and-forth between the two $\mathrm{D}^{+}$ions, until the separation between the two $\mathrm{D}^{+}$ ions becomes too large for the electron to cross or tunnel through the potential energy barrier that separates the two $\mathrm{D}^{+}$ ions. This dynamics can be very well captured by means of semiclassical calculations that describe the laser-driven localization in terms of a passage of the nuclear wave packet through a number of avoided crossings on laser-dressed potential energy curves, which can be very well described by means of a LandauZener formalism. ${ }^{49}$

\section{Towards attosecond pump-probe experiments: observing vibrational wave packets in $\mathrm{H}_{2}{ }^{+}$}

The first molecular pump-probe experiment using attosecond laser pulses was a study where vibrational wave packet dynamics in $\mathrm{H}_{2}{ }^{+}$were observed. An IAP ionized $\mathrm{H}_{2}$, launching a vibrational wave packet in the $1 \mathrm{~s} \sigma_{\mathrm{g}}$ state. At a variable time delay a few-cycle NIR laser was fired, which dissociated the $\mathrm{H}_{2}{ }^{+}$molecular ion, leading to the formation of an $\mathrm{H}^{+}$ion that could be measured, with kinetic energy and angular resolution, using a VMI detector. The dissociation efficiency of the vibrating $\mathrm{H}_{2}{ }^{+}$molecular ion by the NIR pulse strongly favors times when the $\mathrm{H}_{2}{ }^{+}$internuclear distance is stretched, since this minimizes the gap between the $1 \mathrm{~s} \sigma_{\mathrm{g}}$ and the $2 \mathrm{p} \sigma_{\mathrm{u}}$ state across which the excitation occurs. As a result the experiment (see Fig. 8a) allows one to observe at which times the $\mathrm{H}_{2}^{+}$vibrational wave packet is near the outer turning point of the $1 \mathrm{~s} \sigma_{\mathrm{g}}$ potential well. Clear oscillations, including de-phasing and re-phasing of the wave packet, could be observed.

Fig. $8 \mathrm{~b}$ shows a Fourier transform (FT) of the signal that was measured (and simulated, using a TDSE calculation) between 0 and $1.2 \mathrm{eV}$ kinetic energy. This FT shows that the experiment can be understood in terms of number of two-level quantum beats, establishing the connection between attosecond experiments and well-known time-resolved vibrational dynamics as it had previously been observed numerous times in femtochemistry experiments. As a result, the stage was set for the first experiments using attosecond pump-probe techniques to explore electron dynamics on the attosecond timescales.

\section{First attosecond pump-probe experiments: electron localization in dissociative ionization of $\mathrm{D}_{2}$ and $\mathrm{H}_{2}$}

The first successful two-color XUV + NIR experiment combining an IAP to initiate molecular dynamics and a few-cycle NIR pulse to time-dependently probe or control this dynamics was published in ref. 15. In order to successfully perform these experiments, where only about $10^{5}-10^{6}$ XUV photons per pulse were available in the IAP (at $1 \mathrm{kHz}$ ), dedicated improvements in the VMI spectrometer were first needed. Rather than using a molecular target that was generated in a skimmed molecular beam, the gas was introduced by incorporating a small capillary in the repeller electrode of the VMI assembly, which furthermore was given a conical shape, in order to minimize changes to the equipotential lines in the extraction region of the VMI. ${ }^{50}$

In the experiment, an IAP covering a spectral range from 20 to $40 \mathrm{eV}$, obtained by HHG including the use of polarization gating, ${ }^{11,51}$ was used to ionize or excite neutral $\mathrm{H}_{2} / \mathrm{D}_{2}$ molecules. At a variable time delay, a 6 fs NIR pulse with an estimated peak intensity of $6 \times 10^{12} \mathrm{~W} \mathrm{~cm}^{-2}$ interacted with the molecules. Like in the earlier experiment on CEP control of electron localization, ${ }^{33}$ the main experimental observation consisted of a measurement of the kinetic energy-resolved asymmetry of the ejection of $\mathrm{H}^{+} / \mathrm{D}^{+}$ions left or right along the laser polarization axis. The main result of the experiment is shown in Fig. 9a. This figure shows the normalized asymmetry (again defined by eqn (7)) in the ejection of $\mathrm{D}^{+}$ions resulting from twocolor XUV + NIR dissociative ionization of $\mathrm{D}_{2}$, as a function of the XUV-NIR time delay. The main observation is that oscillations occur in the asymmetry over a wide range of kinetic energies and over a wide range of XUV-NIR time delays, including both positive time delays (where the NIR pulse follows the XUV IAP) and the region of time-overlap, where 
a)

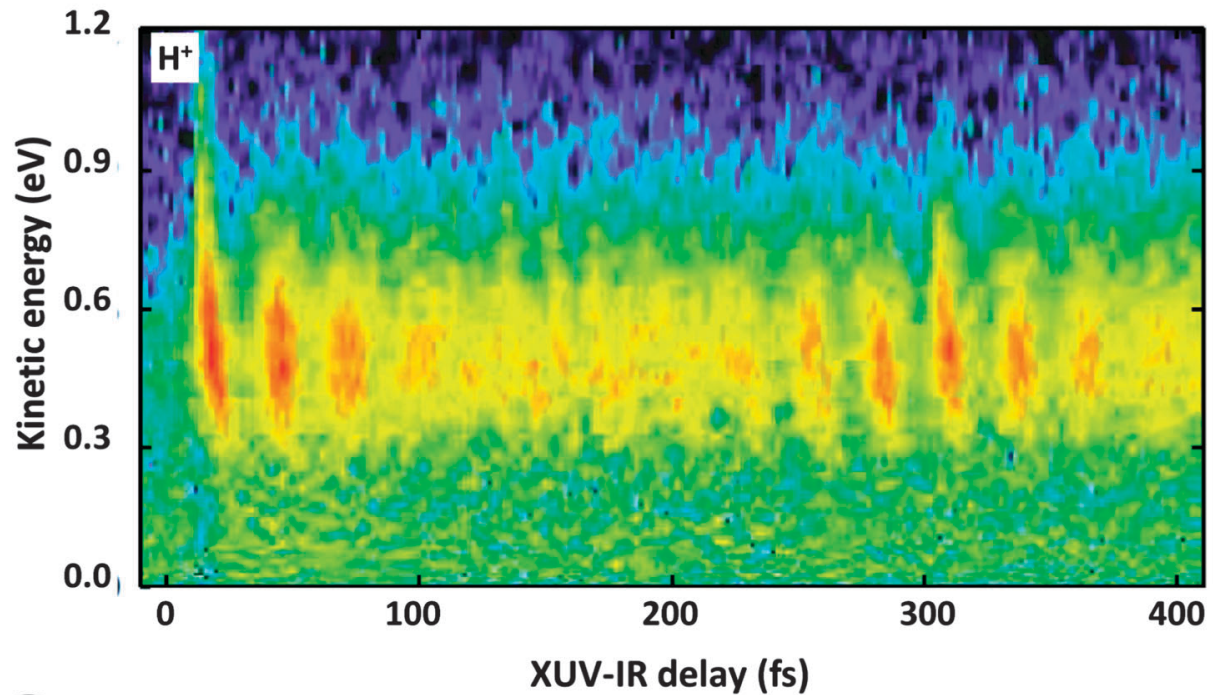

b)

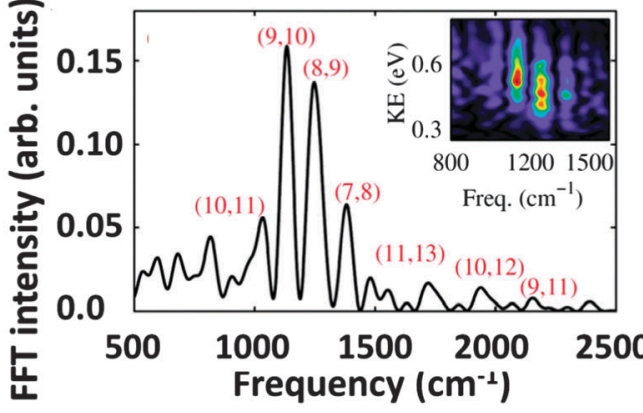

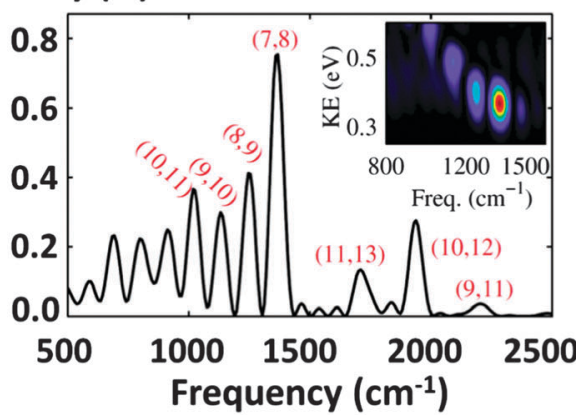

Fig. 8 (a) $\mathrm{H}^{+}$kinetic energy distribution as a function of the delay between an IAP populating the $1 \mathrm{~s} \sigma_{\mathrm{g}}$ ground state of $\mathrm{H}_{2}{ }^{+}$and a few-cycle NIR pulse that dissociates the $\mathrm{H}_{2}{ }^{+}$molecular ion. (b) (left) FFT of the experimental data shown in (a), integrated between 0 and $1.2 \mathrm{eV}$, revealing the contribution from a number of vibrational coherences. The inset shows a kinetic energy-resolved FFT, revealing a correlation between the vibrational coherence and the $\mathrm{H}^{+}$ kinetic energy; (right) equivalent plots, obtained by numerical integration of the TDSE (adapted from ref. 59, copyright APS).

the molecule is ionized or excited in the presence of the NIR field.

A detailed theoretical analysis of the experimental results revealed that the experimental results shown in Fig. 9a are caused by two mechanisms, which are schematically presented in Fig. 9b and c. The first of these mechanisms, shown in Fig. 9b, is very similar to the previously discussed mechanism for CEP control of electron localization, ${ }^{33}$ and relies on an NIR laserdriven coupling between the $2 \mathrm{p} \sigma_{\mathrm{u}}$ and the $1 \mathrm{~s} \sigma_{\mathrm{g}}$ state. The main difference with the work reported in ref. 33 is that the population of the $2 \mathrm{p} \sigma_{\mathrm{u}}$ state does not occur by means of an NIR-driven re-collision process, but by means of single-photon ionization by the XUV IAP (or, possibly, two-color ionization were the XUV excites a doubly excited neutral Rydberg state converging on the $2 \mathrm{p} \sigma_{\mathrm{u}}$ ionic state, which can be ionized by the absorption of a few NIR photons). The XUV-NIR time delay moves the oscillations of the NIR field with respect to the time of ionization and hence influences the location of the NIR laser-induced avoided crossings that the dissociating wave packet encounters on the laserdressed potentials, in much the same way that a variation of the CEP moves these avoided crossings. ${ }^{49}$

However, this mechanism does not explain why electron localization also occurs when the XUV and NIR pulses overlap in time. As revealed by close-coupling calculations, ${ }^{52}$ a second mechanism (shown in Fig. 9c) is responsible for the parity breaking of the wave function in this situation. As before, the challenge is to understand how the $\mathrm{D}^{+}$ion that is detected in the VMI spectrometer results from the formation of a coherent superposition state with both gerade and ungerade components. It turns out that the gerade component is formed by means of an auto-ionization process. The XUV IAP excites the molecule to the neutral doubly excited $\mathrm{Q}_{1}$-states that are located a few eV below the $2 p \sigma_{u}$ ionic state. Like the $2 p \sigma_{u}$ state, the $\mathrm{Q}_{1}$-states are strongly repulsive, and hence the nuclear wave packet rapidly acquires a large kinetic energy. However, as long as the $\mathrm{Q}_{1}$ state lies above the $1 \mathrm{~s} \sigma_{\mathrm{g}}$ ground state, an autoionization process can occur, producing a wave packet on the ground state that dissociates producing $\mathrm{D}^{+}$ions with an appreciable kinetic energy. As before, fast $\mathrm{D}^{+}$ions can be formed along the $2 \mathrm{p} \sigma_{\mathrm{u}}$ curve, as a result of XUV-induced ionization. Hence we can understand that on both the gerade and ungerade curves dissociative wave packets propagate which leads to overlapping kinetic energy distributions, a prerequisite for the observation of electron localization. However, there is still one component missing here. So far, we have only considered the role of the XUV pulse. Following dipole selection rules the overall (ion + photoelectron) wave 

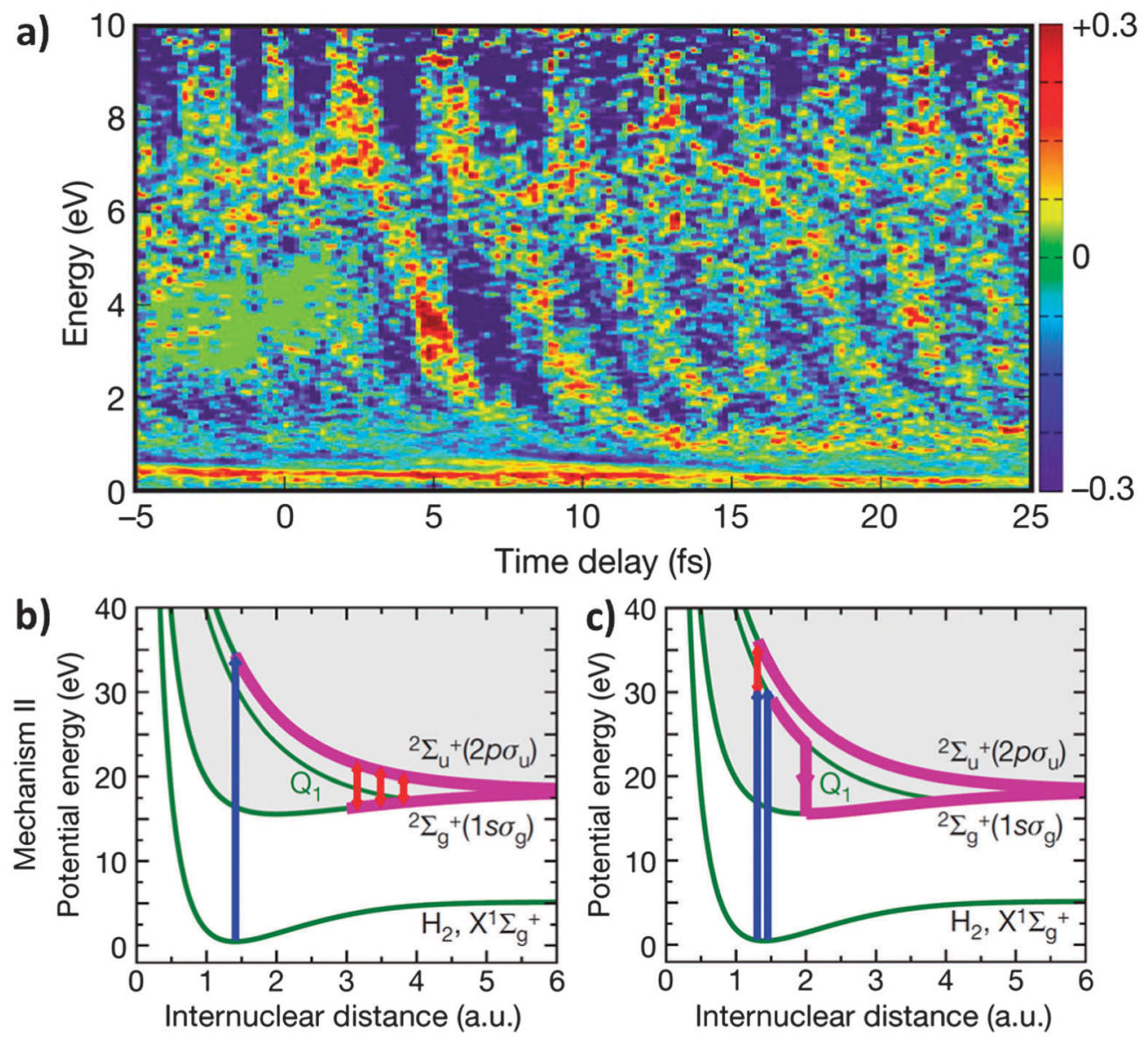

Fig. 9 (a) Time-resolved observation and control of electron localization in a pump-probe experiment, where $\mathrm{H}_{2} / \mathrm{D}_{2}$ molecules were exposed to an XUV IAP and a time-delayed NIR few-cycle laser. $\mathrm{H}^{+} / \mathrm{D}^{+}$fragments resulting from dissociative ionization were recorded using a VMI detector, and a normalized asymmetry parameter $\left[P(E)_{\text {left }}-P(E)_{\text {right }} /\left[P(E)_{\text {left }}+P(E)_{\text {right }}\right]\right.$ was evaluated as a function of the XUV-NIR time delay. The dependence of the asymmetry on the kinetic energy release and the XUV-NIR time delay is caused by two mechanisms that are schematically presented below. (b) Electron localization as a result of laser-driven coupling of the $2 p \sigma_{u}$ and the $1 s \sigma_{g}$ states during the dissociation of the molecular ion that starts out on the $2 p \sigma_{u}$ state. (c) Electron localization caused by coupling of the electronic degrees of freedom, where the action of the NIR laser on the outgoing photoelectron leads to localization of the bound electron that remains behind in the molecular ion (adapted from ref. 15, copyright Nature Publishing Group).

function must be ungerade for both XUV-induced processes, meaning that the two pathways that we have discussed so far are accompanied by a photoelectron described by even-l partial waves in one case (auto-ionization) and by odd-l partial waves in the other. Hence, the two pathways can in principle be distinguished and do not interfere. What makes them interfere in the end is, as in the first mechanism, the NIR pulse, which can alter the angular momentum of the outgoing electron from odd to even, thereby enforcing the electron localization. The intriguing conclusion, drawn from the close-coupling calculations, is therefore that interaction of the NIR field with the outgoing photoelectron controls the localization of the electron that remains bound in the dissociating $\mathrm{D}_{2}{ }^{+}$molecular ion. This mechanism therefore relies on correlation in the two-electron wave function, and shows that attosecond pump-probe experiments may be used to probe correlation and entanglement in multi-electronic wave functions.

We note that since the experiment described in ref. 15, XUV-NIR experiments on electron localization in dissociative ionization of $\mathrm{D}_{2}$ have meanwhile also been carried out with an
APT with a single attosecond pulse per NIR optical cycle, i.e. in the spirit of the "attosecond quantum stroboscope". 53 Although again relying on the coherent preparation of a superposition state containing contributions from both the $2 \mathrm{p} \sigma_{\mathrm{u}}$ and $1 \mathrm{~s} \sigma_{\mathrm{g}}$ states, the mechanism in this experiment was different, namely XUV preparation of a dissociative wave packet on the $1 \mathrm{~s} \sigma_{\mathrm{g}}$ state, interfering with a wave packet on the $2 \mathrm{p} \sigma_{\mathrm{u}}$ state formed by XUV + NIR ionization.

\section{Towards more complex systems: attosecond dynamics in neutral, polyatomic molecules}

In all the molecular attosecond experiments discussed above, the electronic dynamics that decided the outcome of the experiment occurred in doubly excited states above the ionization threshold or in states of the molecular ion. In parallel, there has been an ongoing effort to extend attosecond studies to neutral molecules, through the excitation of valence electron wave packets. ${ }^{54,55}$ An open question that is to be addressed in the coming years is whether the preparation of electronic coherences in molecules (i.e. the coherent preparation of 
electronic superposition states, exploiting the large bandwidth in attosecond laser pulses) allows novel paths towards chemical control that are not available in more traditional experiments that can be fully understood by considering the initial optical excitation to a single, specific Born-Oppenheimer state.

In the last few years we have undertaken several experiments trying to observe attosecond timescale electron dynamics in neutral molecules. Our first attempt was an extension of the $\mathrm{XUV}+\mathrm{NIR}$ attosecond pump-probe experiment on $\mathrm{H}_{2}$ discussed above, where we demonstrated that in dissociative ionization of $\mathrm{H}_{2}$ the $2 \mathrm{p} \sigma_{\mathrm{u}} / 1 \mathrm{~s} \sigma_{\mathrm{g}}$ branching ratio can be controlled on attosecond timescales using a moderately strong NIR laser field. ${ }^{8}$ An APT was used to ionize $\mathrm{H}_{2}$ in the presence of a $3 \times 10^{13} \mathrm{~W} \mathrm{~cm}^{-2}$ NIR laser field, and the $\mathrm{H}^{+}$fragment ion kinetic energy distribution was measured as a function of the XUV-NIR pulse delay, revealing RABBITT-like, sub-NIR-cycle oscillations in the yield of high energy $\mathrm{H}^{+}$fragments. ${ }^{8}$ The remarkable feature in these experiments was that, unlike normal RABBITT, the NIR not only coupled different photoelectron kinetic energies within one and the same ionization continuum, but moreover provided a coupling between the $2 \mathrm{p} \sigma_{\mathrm{u}}$ and $1 \mathrm{~s} \sigma_{\mathrm{g}}$ continua, thereby providing access to control over the $2 \mathrm{p} \sigma_{\mathrm{u}} / 1 \mathrm{~s} \sigma_{\mathrm{g}}$ branching ratio. However, having stated this, it is clear that there is no role in this explanation of the experiment for time-dependent electron dynamics in the neutral molecule (i.e. prior to ionization). A similar conclusion was reached in experiments on $\mathrm{O}_{2}$ that were carried out under similar conditions, where $\mathrm{O}^{+}$fragment kinetic energy distributions could be controlled as well by means of the XUV-NIR delay. ${ }^{56}$

We have managed only very recently to obtain the first compelling evidence for attosecond timescale electron dynamics in neutral molecules. ${ }^{34}$ In these experiments a series of small molecules $\left(\mathrm{N}_{2}, \mathrm{CO}_{2}\right.$ and $\left.\mathrm{C}_{2} \mathrm{H}_{4}\right)$ were ionized by an APT, in the presence of a moderately strong (appr. $10^{13} \mathrm{~W} \mathrm{~cm}^{-2}$ ) NIR laser field. Rather than using VMI to measure the kinetic energy and angular distribution of ionic fragments resulting from dissociative ionization, the experiment monitored the total ionization yield (i.e. parent + fragment ions) as a function of XUV-NIR delay, leading to the clear conclusion (supported by TDDFT calculations) that the oscillatory electric field of the NIR laser can modulate the ionization efficiency by the APT in an oscillatory manner. Simply put, the NIR electric field polarizes the molecules, inducing time-dependent density variations that affect the probability that an XUV photon can be absorbed. In future, it is intended to extend these experiments to aligned molecules, ${ }^{57,58}$ and to extend the attosecond probing of timedependent electron density variations to perturbative dynamics, i.e. coherent superposition states that persist even in the absence of a driving NIR field.

\section{Conclusion and outlook}

In this review, an overview of the recent development of attosecond science has been presented, emphasizing in particular the role that a series of velocity map imaging (VMI) experiments have played in the initial characterization of attosecond pulse trains (APTs) ${ }^{30}$ and isolated attosecond pulses (IAPs), ${ }^{38}$ as well as in a number of application experiments that have been performed in both atomic and molecular systems. ${ }^{7,14,15,31,59}$ As illustrated by these examples, the multidimensional nature of VMI photoelectron/photo-ion detection, allowing collection of charged particles with unit detection efficiency and with both kinetic energy and angular resolution, matched very well with the demands of these early attosecond experiments. Consequently, VMI spectrometers have become a standard tool in many attosecond laboratories, as well as, more generally, laboratories using intense laser fields. Beyond the work described in this review, where we have emphasized experiments aimed at exploiting the attosecond resolution provided by XUV/X-rays generated by high-harmonic generation (HHG), VMI spectrometers are finding widespread use in experiments where XUV/X-rays (both generated by HHG and obtained from recently developed XUV/X-ray free electron lasers) are used to study multi-photon ionization or timedependent molecular dynamics exploiting the high kinetic energy of photoelectrons ejected by an XUV/X-ray photon. ${ }^{60-64}$

Let us consider the future of the use of VMI detection in attosecond experiments. As demonstrated by this review, successfully performing attosecond pump-probe experiments on simple atomic or molecular systems with VMI detection of the resulting photoelectrons or (fragment) ions has become possible in the last few years. However, the successful experiments surveyed in this review mask the fact that uncorrelated observation with a VMI spectrometer of photoelectrons or (fragment) ions in two-color XUV-NIR experiments often leads to data sets that are extremely challenging to interpret. Indeed, while we have been able to obtain insightful experimental outcomes for simple systems like $\mathrm{H}_{2}$, the analysis of attosecond experiments on small molecules like $\mathrm{O}_{2}, \mathrm{~N}_{2}$ or $\mathrm{CO}_{2}$ has proven to be very tough, due to the participation of many electronic states and the non-perturbative nature of the NIR interaction. Interpretation of these experiments has thus far only been possible through very intensive collaboration with theoretical groups. As experimentalists, the challenge thus becomes to perform more insightful experiments, an obvious path being the extension of attosecond XUV + NIR experiments to correlated detection of the full 3D momenta of both photoelectrons and fragment ions, using a reaction microscope. ${ }^{43-45}$ Such experiments are starting up in several laboratories, and are likely to replace VMI spectrometers in attosecond experiments on simple systems.

The situation is likely to be somewhat different when considering attosecond experiments on more complicated systems, such as small bio-molecules. On the one hand, study of these systems using reaction microscopes appears less tractable, due to - among other things - the breakdown of the axial recoil approximation, while on the other hand, insightful information may likely be obtained through simpler observables, such as the kinetic energy and angular distribution of fragment ions. Already, a first study has been published where charge migration in phenyl-aniline was monitored using time-of-flight mass 
spectroscopy, ${ }^{65}$ and we may anticipate that the use of velocity map ion imaging will provide significant further insight into this situation, or into related experiments that have recently been performed.

A very prominent role for VMI is to be expected in future XUV IAP pump-XUV IAP probe experiments. Given the fact that these experiments necessarily rely on very high attosecond pulse intensities, i.e. low repetition rate lasers, the use of VMI detection promises to be an appropriate method to maximize the information content that is extracted from the experiments.

The attosecond science field is still very young, and hence "attosecond imaging" may still have many surprises in store that we cannot foresee at the moment. But it appears clear that the combination of attosecond pump-probe spectroscopy with VMI detection is a powerful one, where important results are likely to be obtained in the years to come.

\section{Acknowledgements}

The material presented in this review is to a large extent based on collaborative work presented in ref. 7, 14, 15, 30, 31, 38 and 59. In this context I would particularly like to acknowledge our fruitful collaborations with Prof. Anne L'Huillier and Dr Johan Mauritsson (Univ. of Lund), Prof. Ken Schafer (LSU), Profs Giuseppe Sansone and Mauro Nisoli (Politecnico di Milano), Profs Matthias Kling and Ferenc Krausz (MPQ-Garching), Prof. Fernando Martin (Univ. Autonoma Madrid), Prof. Misha Ivanov (MBI), and a number of previous PhD students and postdocs in my team, in particular Dr Sergey Aseyev, Dr Freek Kelkensberg, Dr Wing Kiu Siu, Dr Omair Ghafur, Dr Tatiana Martchenko, Dr Julia Jungmann and Dr Arjan Gijsbertsen. I thank Dr Johan Mauritsson (Univ. of Lund) for his permission to use Fig. 5 (taken from ref. 32).

\section{References}

1 A. H. Zewail, Femtochemistry: Atomic-Scale Dynamics of the Chemical bond using ultrafast lasers - (Nobel lecture), Angew. Chem., Int. Ed., 2000, 39, 2587.

2 F. Krausz and M. Ivanov, Attosecond physics, Rev. Mod. Phys., 2009, 81, 163.

3 H. Niikura and P. B. Corkum, Attosecond and Angstrom science, Adv. At., Mol., Opt. Phys., 2007, 54, 511.

4 P. Agostini and L. F. DiMauro, The physics of attosecond light pulses, Rep. Prog. Phys., 2004, 67, 813.

5 P. B. Corkum, Plasma Perspective on Strong-Field Multiphoton Ionization, Phys. Rev. Lett., 1993, 71, 1994.

6 P. M. Paul, et al., Observation of a train of attosecond pulses from high harmonic generation, Science, 2001, 292, 1689.

7 T. Remetter, et al., Attosecond electron wave packet interferometry, Nat. Phys., 2006, 2, 323.

8 F. Kelkensberg, et al., Attosecond Control in Photoionization of Hydrogen Molecules, Phys. Rev. Lett., 2011, 107, 043002.

9 M. Hentschel, et al., Attosecond metrology, Nature, 2001, 414, 509.
10 R. Kienberger, et al., Steering attosecond electron wave packets with light, Science, 2002, 297, 1144.

11 G. Sansone, et al., Isolated single-cycle attosecond pulses, Science, 2006, 314, 443.

12 H. Mashiko, et al., Double optical gating of high-order harmonic generation with carrier-envelope phase stabilized lasers, Phys. Rev. Lett., 2008, 100, 103906.

13 M. J. Abel, et al., Isolated attosecond pulses from ionization gating of high-harmonic emission, Chem. Phys., 2009, 366, 9.

$14 \mathrm{~J}$. Mauritsson, et al., Attosecond Electron Spectroscopy Using a Novel Interferometric Pump-Probe Technique, Phys. Rev. Lett., 2010, 105, 053001.

15 G. Sansone, et al., Electron localization following attosecond molecular photoionization, Nature, 2010, 465, 763.

16 A. McPherson, et al., Studies of multiphoton production of vacuum-ultraviolet radiation in the rare gases, J. Opt. Soc. Am. B, 1987, 4, 595.

17 M. Ferray, et al., Multiple-harmonic conversion of $1064 \mathrm{~nm}$ radiation in rare gases, J. Phys. B: At., Mol. Opt. Phys., 1988, 21, L31.

18 P. Tzallas, et al., Generation of intense continuum extremeultraviolet radiation by many-cycle laser fields, Nat. Phys., 2007, 3, 846.

$19 \mathrm{Y} . \mathrm{Wu}$, et al., Generation of high-flux attosecond extreme ultraviolet continuum with a 10 TW laser, Appl. Phys. Lett., 2013, 102, 201104.

20 D. Herrmann, et al., Generation of sub-three-cycle, 16 TW light pulses by using noncollinear optical parametric chirped-pulse amplification, Opt. Lett., 2009, 34, 2459.

21 P. Tzallas, E. Skantzakis, L. A. A. Nikolopoulos, G. D. Tsakiris and D. Charalambidis, Extreme-ultraviolet pumpprobe studies of one-femtosecond-scale electron dynamics, Nat. Phys., 2011, 7, 781.

22 E. Goulielmakis, et al., Real-time observation of valence electron motion, Nature, 2010, 466, 739.

23 C. Ott, et al., Lorentz Meets Fano in Spectral Line Shapes: A Universal Phase and Its Laser Control, Science, 2013, 340, 716.

24 R. Kienberger, et al., Sub-femtosecond X-ray pulse generation and measurement, Appl. Phys. B: Lasers Opt., 2002, 74, S3.

25 R. Kienberger, et al., Atomic transient recorder, Nature, 2004, 427, 817.

26 Y. Mairesse and F. Quere, Frequency-resolved optical gating for complete reconstruction of attosecond bursts, Phys. Rev. A, 2005, 71, 011401.

27 M. Drescher, et al., Time-resolved atomic inner-shell spectroscopy, Nature, 2002, 419, 803.

28 P. Kruit and F. H. Read, Magnetic field paralleliser for 2? electron-spectrometer and electron-image magnifier, J. Phys. E: Sci. Instrum., 1983, 16, 313.

29 A. T. J. B. Eppink and D. H. Parker, Velocity map imaging of ions and electrons using electrostatic lenses: application in photoelectron and photofragment ion imaging of molecular oxygen, Rev. Sci. Instrum., 1997, 68, 3477.

30 S. A. Aseyev, Y. Ni, L. J. Frasinski, H. G. Muller and M. J. J. Vrakking, Attosecond angle-resolved photoelectron spectroscopy, Phys. Rev. Lett., 2003, 91, 223902. 
31 K. Varju, et al., Angularly resolved electron wave packet interferences, J. Phys. B: At., Mol. Opt. Phys., 2006, 39, 3983.

32 J. Mauritsson, et al., Coherent electron scattering captured by an attosecond quantum stroboscope, Phys. Rev. Lett., 2008, 100, e73003.

33 M. F. Kling, et al., Control of electron localization in molecular dissociation, Science, 2006, 312, 246.

34 C. Neidel, et al., Probing Time-Dependent Molecular Dipoles on the Attosecond Time Scale, Phys. Rev. Lett., 2013, 111, 033001.

35 H. G. Muller, Reconstruction of attosecond harmonic beating by interference of two-photon transitions, Appl. Phys. B: Lasers Opt., 2002, 74, S17.

36 E. S. Toma and H. G. Muller, Calculation of matrix elements for mixed extreme-ultraviolet-infrared two-photon abovethreshold ionization of argon, J. Phys. B: At., Mol. Opt. Phys., 2002, 35, 3435.

37 E. S. Toma, P. Antoine, A. de Bohan and H. G. Muller, Resonance-enhanced high-harmonic generation, J. Phys. B: At., Mol. Opt. Phys., 1999, 32, 5843.

38 S. Zherebtsov, et al., Attosecond imaging of XUV-induced atomic photoemission and Auger decay in strong laser fields, J. Phys. B: At., Mol. Opt. Phys., 2011, 44, 105601.

39 P. A. Korneev, et al., Interference Carpets in Above-Threshold Ionization: From the Coulomb-Free to the Coulomb-Dominated Regime, Phys. Rev. Lett., 2012, 108, 223601.

40 Y. Huismans, et al., Photoelectron angular distributions from the ionization of xenon Rydberg states by midinfrared radiation, Phys. Rev. A, 2013, 87, 033413.

41 K. Klünder, et al., Reconstruction of attosecond electron wave packets using quantum state holography, Phys. Rev. A, 2013, 88, 033404.

42 S. Haessler, et al., Phase-resolved attosecond near-threshold photoionization of molecular nitrogen, Phys. Rev. A, 2009, 80, 011404.

$43 \mathrm{~J}$. Ullrich, et al., Recoil-ion and electron momentum spectroscopy: reaction-microscopes, Rep. Prog. Phys., 2003, 66, 1463.

44 A. Fischer, et al., Electron Localization Involving Doubly Excited States in Broadband Extreme Ultraviolet Ionization of H_\{2\}, Phys. Rev. Lett., 2013, 110, 213002.

45 F. Furch, et al., Carrier-envelope phase stable few-cycle pulses at $400 \mathrm{kHz}$ for electron-ion coincidence experiments, Opt. Express, 2013, 21, 22671.

46 I. Znakovskaya, et al., Attosecond Control of Electron Dynamics in Carbon Monoxide, Phys. Rev. Lett., 2009, 103, 103002.

47 A. Baltuska, et al., Attosecond control of electronic processes by intense light fields, Nature, 2003, 421, 611.

48 G. G. Paulus, et al., Absolute-phase phenomena in photoionization with few-cycle laser pulses, Nature, 2001, 414, 182.

49 F. Kelkensberg, G. Sansone, M. Y. Ivanov and M. Vrakking, A semi-classical model of attosecond electron localization in dissociative ionization of hydrogen, Phys. Chem. Chem. Phys., 2011, 13, 8647.

50 O. Ghafur, et al., A velocity map imaging detector with an integrated gas injection system, Rev. Sci. Instrum., 2009, 80, 033110.

51 I. J. Sola, et al., Controlling attosecond electron dynamics by phase-stabilized polarization gating, Nat. Phys., 2006, 2, 319.

52 J. L. Sanz-Vicario, H. Bachau and F. Martin, Timedependent theoretical description of molecular autoionization produced by femtosecond xuv laser pulses, Phys. Rev. A, 2006, 73, 033410.

53 K. P. Singh, et al., Control of Electron Localization in Deuterium Molecular Ions using an Attosecond Pulse Train and a Many-Cycle Infrared Pulse, Phys. Rev. Lett., 2010, 104, e023001.

54 F. Remacle, M. Nest and R. D. Levine, Laser Steered Ultrafast Quantum Dynamics of Electrons in LiH, Phys. Rev. Lett., 2007, 99, 183902.

55 B. Mignolet, A. Gijsbertsen, M. J. J. Vrakking, R. D. Levine and F. Remacle, Stereocontrol of attosecond time-scale electron dynamics in ABCU using ultrafast laser pulses: a computational study, Phys. Chem. Chem. Phys., 2011, 13, 8331.

56 W. Siu, et al., Attosecond control of dissociative ionization of O_\{2\} molecules, Phys. Rev. A, 2011, 84, 063412.

57 F. Rosca-Pruna and M. J. J. Vrakking, Experimental observation of revival structures in picosecond laser-induced alignment of I-2, Phys. Rev. Lett., 2001, 87, 153902.

58 H. Stapelfeldt and T. Seideman, Colloquium: Aligning molecules with strong laser pulses, Rev. Mod. Phys., 2003, 75, 543.

59 F. Kelkensberg, et al., Molecular Dissociative Ionization and Wave-Packet Dynamics Studied Using Two-Color XUV and IR Pump-Probe Spectroscopy, Phys. Rev. Lett., 2009, 103, 123005.

60 P. Johnsson, et al., Velocity map imaging of atomic and molecular processes at the free electron laser in Hamburg (FLASH), J. Mod. Opt., 2008, 55, 2693.

$61 \mathrm{~N}$. Berrah, et al., Non-linear processes in the interaction of atoms and molecules with intense EUV and X-ray fields from SASE free electron lasers (FELs), J. Mod. Opt., 2010, 57, 1015.

62 P. Johnsson, et al., Characterization of a two-color pump? probe setup at FLASH using a velocity map imaging spectrometer, Opt. Lett., 2010, 35, 4163.

63 F. Kelkensberg, et al., XUV ionization of aligned molecules, Phys. Rev. A, 2011, 84, 051404.

64 A. Rouzée, et al., Towards imaging of ultrafast molecular dynamics using FELs, J. Phys. B: At., Mol. Opt. Phys., 2013, 46, 164029.

65 L. Belshaw, et al., Observation of Ultrafast Charge Migration in an Amino Acid, J. Phys. Chem. Lett., 2012, 3, 3751. 